\title{
Clinicopathological analysis of epithelioid inflammatory myofibroblastic sarcoma
}

\author{
XUEMEI DU ${ }^{1}$, YING GAO $^{1}$, HONGYU ZHAO $^{1},{\text { BIN } \mathrm{LI}^{2}, \text { WEIMIN XUE }^{3} \text { and DAYE WANG }}^{4}$ \\ Departments of ${ }^{1}$ Pathology and ${ }^{2}$ Nuclear Magnetic Resonance, Capital Medical University Affiliated Beijing \\ Shijitan Hospital, Beijing 100038; ${ }^{3}$ Cyttel Biosciences Inc., Beijing 101111; ${ }^{4}$ Department of Pathology, \\ Basic Medical College, Capital Medical University, Beijing 100069, P.R. China
}

Received May 19, 2017; Accepted February 12, 2018

DOI: $10.3892 / \mathrm{ol} .2018 .8530$

\begin{abstract}
Inflammatory myofibroblastic tumor (IMT) is a distinctive neoplasm composed of myofibroblastic and fibroblastic spindle cells, accompanied by the inflammatory infiltration of plasma cells, lymphocytes and/or eosinophils. Epithelioid inflammatory myofibroblastic sarcoma(EIMS), which primarily consists of cells with a round or epithelioid morphology, is associated with a poor prognosis and rapid development of local recurrence, and has been recognized to be a variant of IMT. Diagnosis of EIMS is difficult owing to its close resemblance to malignant mesothelioma, anaplastic large cell lymphoma, gastrointestinal stromal tumor and other malignant diseases. In the present study, a case of this rare tumor was evaluated in a 26-year-old male who was admitted to hospital after experiencing abdominal pain for $\sim 18$ days and abdominal distention for 1 week. The patient's tumor was examined by imaging, gross examination, histology, immunohistochemistry and fluorescence in situ hybridization (FISH). The magnetic resonance imaging enhanced-scanning image revealed that the morphology of the tumor was irregular, and signal was medley consisting of high and low hybrid reinforcement. Tumors were located in the bladder and rectal pit, in the lower part of the lower abdomen, indicating the presence of malignancy and involvement of the small intestine and rectum. Enhanced-scanning imaging revealed notable inhomogeneous enhancement. Gross examination revealed that the tumor was solid and had a variegated appearance with alternating fleshy and mucoid areas in the cut surface. Microscopically, the tumors were dominated by sheets of epithelioid-to-round cells with a prominent inflammatory infiltrate. The majority of the stroma was myxoid. Immunohistochemically, the tumor cells exhibited diffuse strong staining for ALK receptor tyrosine kinase (hereafter ALK), vimentin, tumor protein P53, desmin, Wilms' tumor
\end{abstract}

Correspondence to: Dr Daye Wang, Department of Pathology, Basic Medical College, Capital Medical University, 10 Xitoutiao, You An Men, Beijing 100069, P.R. China

E-mail: wangdaye@ccmu.edu.cn

Key words: epithelioid inflammatory myofibroblastic sarcoma, programmed cell-death ligand 1 , immunohistochemistry, therapy
1 and programmed death-ligand 1. FISH analysis also revealed the existence of ALK rearrangement. The expression of PD-L1 in EIMS indicates that the immune checkpoint blockade could represent a novel therapy for the treatment of EIMS.

\section{Introduction}

Inflammatory myofibroblastic tumor (IMT) is a mesenchymal neoplasm composed of myofibroblastic spindle cells in a myxoid to collagenous stroma with an inflammatory infiltrate chiefly comprised of plasma cells and lymphocytes, occasionally admixed with eosinophils and neutrophils (1). IMT usually affects children and adolescents, although a broad age range has been documented. The most common anatomical locations are the abdominopelvic region, lung, mediastinum and retroperitoneum (1).

In a mesenteric inflammatory myofibroblastic tumor, T1 weighted sequence four minutes after Gadolinium injection demonstrated intense enhancement of the peripheral inflammatory component, whilst the central fibrotic component is hypovascular (2). IMT is considered to be a soft tissue tumor with an intermediate biological behavior. However, a small percentage of cases behave aggressively (3).

Epithelioid inflammatory myofibroblastic sarcoma (EIMS) is considered to be a variant of inflammatory myofibroblastic tumor (IMT), as it has similar malignant characteristics and mainly consists of round-to-epithelioid cells (4) with a pattern of nuclear membrane or perinuclear immunostaining for ALK receptor tyrosine kinase (hereafter ALK). ALK is a receptor tyrosine kinase gene located on chromosome $2 \mathrm{p} 23$. Rearrangements involving this gene generally result in hyperactivity of ALK protein and correlate well with ALK protein expression by immunohistochemistry (5). Subsequent studies further demonstrated that this distinctive nuclear membrane staining pattern of ALK corresponded to ALK-RANBP2 fusion (6-8). The nuclear membrane staining pattern of ALK seems to be suggestive of RANBP2-ALK fusion in IMT because it has not been observed in other ALK rearrangements to date. The RANBP2 gene, located on chromosome $2 q 12$, encodes a 358-kd nuclear pore protein and, therefore, has been attributed to the nuclear membranous localization of ALK expression by immunostaining (6,7). Clinically, EIMS is more clinically aggressive than IMT and patients exhibit 
reduced disease-free survival rates (4). Recognizing EIMS as a distinct variant of IMT is very important as patients with ALK-rearrangement EIMS may benefit from targeted therapy. EIMS closely resembles malignant mesothelioma (MM), anaplastic large cell lymphoma (ALCL), gastrointestinal stromal tumor (GIST) and other cancer types, meaning it is difficult to diagnose. Magnetic resonance imaging (MRI) enhanced scanning is a MRI scan following an intravenous injection of a contrast agent. This is a useful clinical technique for evaluating the severity, location and extent of the tumor (9). As EIMS has not been widely recognized, we analyzed its imaging, clinicopathological, immunohistochemical, molecular cytogenetics and treatment. The aim of the present study was to improve understanding of the disease.

\section{Materials and methods}

Patient information and selection. A 26-year-old male patient diagnosed with EIMS, who had been in abdominal pain for eighteen days and abdominal distention for 1 week, presented to the Capital Medical University Affiliated Beijing Shijitan Hospital (Beijing, China) in November 2015. This patient was selected for the present study as they presented with inflammatory myofibroblastic tumor cells with a round or epithelial morphology. The present study was approved by ethics committee of the Capital Medical University Affiliated Beijing Shijitan Hospital (Beijing, China) and the patient provided written informed consent for inclusion.

Imaging examination. The lesion was observed with magnetic resonance imaging enhanced scanning at the Capital Medical University Affiliated Beijing Shijitan Hospital (Beijing, China). A MRI scan was carried out following intravenous injection of some kind of contrast agent Gd-DTPA $(0.2 \mathrm{ml} / \mathrm{kg}$, Consun Pharmaceutical Group Limited, Guangzhou, China). Dynamic contrast-enhanced MRI has become an important component of the multiparametric strategy and is emerging as a useful clinical technique for evaluating the severity, location and extent of the tumor (9).

Histopathological analysis. Removed surgical specimens were fixed in 10\% phosphate-buffered, neutral formaldehyde solution at room temperature for $24 \mathrm{~h}$ and dehydrated in an ascending series of ethanol. Samples were routinely embedded in paraffin, washed with xylene, then rehydrated in a descending series of alcohol, washed with distilled water, and stained with hematoxylin and eosin for $30 \mathrm{~min}$ at room temperature. Sections (4- $\mu \mathrm{m}$ thick) were observed under a light microscope with the magnifications of x40, x100, x200 and x400.

Immunohistochemical study. Removed surgical specimens were fixed in $10 \%$ phosphate-buffered, neutral formaldehyde solution at room temperature for $24 \mathrm{~h}$. Tissue sections $(4-\mu \mathrm{m}$ thick) were deparaffinized, rehydrated and antigen retrieval with working solution of EnVision, FLEX Target Retrieval solution High $\mathrm{Ph}$ (50x) according to the manufacturer's protocol [EnVision FLEX+, Mouse, high Ph (Link) HRP; cat. no. K8002; Dako; Agilent Technologies, Inc., Santa Clara, CA, USA] in PT Link (cat. no. PT100; Dako; Agilent Technologies, Inc.) at $95^{\circ} \mathrm{C}$ for $20 \mathrm{~min}$, and washed in distilled water (10).
Endogenous peroxidase was blocked by DAKO Envision flex peroxidase- blocking reagent for $10 \mathrm{~min}$, then washed again three times in the EnVision ${ }^{\mathrm{TM}}$ FLEX Wash buffer (Dako; Agilent Technologies, Inc.). The slides were incubated for 20-30 mins at room temperature in humidity chamber with appropriate dilutions of primary antibodies (primary antibodies are detailed in Table I) along with their positive and negative controls. Immunohistochemistry was done manually according to DAKO EnVision method (10), The sections (4- $\mu \mathrm{m}$ thick) were then incubated with secondary antibody (EnVision FLEX/HRP, cat. no. K8002; Dako; Agilent Technologies, Inc., Santa Clara, CA, USA) for coupling reaction for 20-30 min at room temperature. The substrate (EnVision FLEX DAB+ Chromogen) was used to produce crisp brown color at the site of target antigen. The hematoxylin (1-2 dips) was used as a counter stain. Sections were observed under a light microscope with the magnifications of 40, 100, 200 and $\times 400$.

Fluorescence in situ hybridization (FISH). FISH was performed on $4 \mu \mathrm{m}$-thick paraffin sections with Vysis ALK Break Apart FISH Probe kit, according to the manufacturer's protocol (Abbott Laboratories, Chicago, IL, USA). The probe of ALK dual color separation of Abbott Company are composed of 3 'one ALK orange fluorescent probe $\sim 300 \mathrm{~KB}$ and 5 'one ALK green fluorescent probe $\sim 442 \mathrm{~KB}$. The hybridization results were viewed by an epi-illumination fluorescence microscope (maginification, $\mathrm{x} 1,000)$. For no ALK rearrangement, these specimens contain fused orange and green signals or a single green signal without a corresponding orange signal; whilst for ALK rearrangement, these nuclei contained rearranged or 'broken apart' signals, 2 or more signal diameters apart.

Reverse transcription-polymerase chain reaction (RT-PCR). RT-PCR analysis was performed to investigate the fusion location of the RAN binding protein 2 (RANBP2) and ALK gene. Total RNA was extracted from $20 \mu \mathrm{m}$-thick paraffin sections (AmoyDx ${ }^{\circledR}$ FFPE DNA and RNA Extraction kits; Amoy Diagnostics Co., Ltd., Xiamen, China), and reverse transcription (cat. no. ADx-AE01; Amoy Diagnostics Co., Ltd.) was conducted by random hexamer primers method to cDNA. The primer sequences of RANBP2-ALK were as follows: Forward, 5'-CAGACTCAGTGCCTGATGGATA-3' and reverse, 5'-CGGAGCTTGCTCAGCTTGTA-3'. The PCR (2X Taq Master mix, Takara Bio, Inc., Otsu, Japan) reaction ran for 35 cycles under the following conditions: $95^{\circ} \mathrm{C}$ for 30 sec, $50^{\circ} \mathrm{C}$ for $30 \mathrm{sec}$ and $72^{\circ} \mathrm{C}$ for $60 \mathrm{sec}$. An expected 139-bp amplified product was detected in the present case. $\beta$-actin was included as a reference gene, the primer sequences of $\beta$-actin were as follows: Forward, 5'-CACAGTAGGTCTGAACAG ACTC-3', and reverse, 5'-AGTGATCTCCTTCTGCATCCT G-3'. RANBP2-ALK fusion point was confirmed by direct sequencing (YMFX Biotech Co., Ltd.) of the chimeric cDNA product. The $2^{-\Delta \Delta C q}$ method (11) was used to determine the relative quantification of $\mathrm{miR}$ expression in the tissue samples.

\section{Results}

Magnetic resonance imaging examination. Images from magnetic resonance imaging enhanced scanning demonstrated that the morphology of the tumor was irregular, and 
Table I. Primary antibodies used for immunohistochemistry.

\begin{tabular}{|c|c|c|c|c|}
\hline Target & Supplier & Catalog number & Dilution & Staining \\
\hline ALK & Origene Technologies, Inc., Beijing, China & TA801287 & Ready to use & + \\
\hline PD-L1 & Origene Technologies, Inc. & TA507087 & Ready to use & + \\
\hline PD-1 & Origene Technologies, Inc. & TA314461 & Ready to use & Lymphocyte + \\
\hline Ki-67 & Origene Technologies, Inc. & UM870033 & $1: 100$ & Index $40 \%$ \\
\hline P53 & Gene Tech Co., Ltd., Shanghai, China & GM700101 & Ready to use & + \\
\hline DES & Origene Technologies, Inc. & TA502328 & $1: 60$ & Focally + \\
\hline CK & Origene Technologies, Inc. & ZM-0069 & $1: 80$ & Focal + \\
\hline EMA & Gene Tech Co., Ltd. & GM061329 & $1: 200$ & - \\
\hline CAM5.2 & Origene Technologies, Inc. & ZM-0316 & Ready to use & Focal + \\
\hline Myoglobin & Origene Technologies, Inc. & ZA-0192 & Ready to use & - \\
\hline CALDES & Gene Tech Co., Ltd. & GM355701 & Ready to use & - \\
\hline Vimentin & Gene Tech Co., Ltd. & GM072529 & $1: 120$ & + \\
\hline CD30 & Gene Tech Co., Ltd. & GM075129 & $1: 35$ & - \\
\hline CD3 & Origene Technologies, Inc. & ZM-0417 & $1: 120$ & Lymphocyte + \\
\hline CD20 & Gene Tech Co., Ltd. & GM075529 & $1: 200$ & $\begin{array}{l}\text { Little } \\
\text { lymphocyte + }\end{array}$ \\
\hline CD4 & Origene Technologies, Inc. & ZM-0418 & Ready to use & Lymphocyte + \\
\hline CD8 & Gene Tech Co., Ltd. & GT211202 & Ready to use & Lymphocyte + \\
\hline $\mathrm{MC}$ & Gene Tech Co., Ltd. & ZM-0386 & Ready to use & - \\
\hline Calretinin & Origene Technologies, Inc. & TA353630 & $1: 60$ & Focal + \\
\hline WT-1 & Gene Tech Co., Ltd. & GM356102 & Ready to use & + \\
\hline D2-40 & Gene Tech Co., Ltd. & GM361929 & $1: 60$ & - \\
\hline HMB45 & Origene Technologies, Inc. & ZM-0187 & Ready to use & - \\
\hline CD117 & Origene Technologies, Inc. & ZA-0523 & Ready to use & - \\
\hline DOG1 & Origene Technologies, Inc. & ZM-0371 & Ready to use & - \\
\hline SMA & Origene Technologies, Inc. & ZM-0003 & Ready to use & - \\
\hline
\end{tabular}

CALDES, caldesmon; CK, cytokeratins; DES, desmin; EMA, epithelial membrane antigen; MYF4, myogenin; PD, programmed death; PD-L1, programmed death-ligand 1; CD, cluster of differentiation; MC, mesothelial cells; WT-1, Wilms' tumor 1; DOG1, anoctamin-1; SMA, smooth muscle actin.

signal was medley consisting of high and low hybrid reinforcement. Tumors located in the bladder and rectal pit in the lower part of the lower abdomen indicated the malignancy and involvement of the small intestine and rectum (Fig. 1A). Inhomogeneity was observed following enhanced scanning imaging (Fig. 1B).

Gross examination. Gross examination revealed that the tumor was located in the right abdominal wall $(5.0 \times 3.2 \times 0.5 \mathrm{~cm})$, left iliac fossa $(2.5 \times 2.0 \times 1.0 \mathrm{~cm})$, the greater omentum $(17 \times 12 \times 4.5 \mathrm{~cm})$, part of the right hemi-colon $(1.8 \times 1.5 \times 1.5-0.3 \times 0.3 \times 0.2 \mathrm{~cm})$, the rectum $(5.6 \times 3.5 \times 4.0 \mathrm{~cm})$ and the abdominal cavity $(25 \times 19 \times 10 \mathrm{~cm})$. The tumor was solid and had a variegated appearance, with alternating fleshy and mucoid areas at the cut surface (Fig. 2).

Histopathological examination. Microscopically, tumor cells were not densely arranged. (Fig. 3A), and stroma is myxoid (Fig. 3B). Lymphocytes infiltrated into the stroma (Fig. 3C). The tumor cells were epithelioid, with eccentric nuclei, prominent nucleoli and abundant eosinophilic cytoplasm (Fig. 3D).
Immunohistochemical examination. Immunohistochemical staining results are depicted in Table I. The tumor cells exhibited diffuse strong staining for ALK (Fig. 4A), DES (Fig. 4B), PDL1 (Fig. 4C) and vimentin. Similarly, there was positive staining for Wilms' tumor 1 and tumor protein P53. The Ki-67 index was $\sim 40 \%$. The tumor cells also exhibited positive staining for the cytokeratins (CK) CAM5.2 and calretinin, although this staining was weaker. There was no reactivity to epithelial membrane antigen (EMA), smooth muscle actin (SMA), anoctamin-1 (DOG1), CD117, antibody HMB-45, antibody D2-40, mesothelial cells (MC), CD30, caldesmon or myoglobin. Infiltrated lymphocytes were mainly $\mathrm{T}$ cells, with a small number of B cells also present.

FISH analysis. FISH analysis revealed the presence of ALK rearrangements through the identification of a set of separate green and orange signals, a fused signal in tumor cell nuclei or through only one orange signal and a fused signal (Fig. 4D).

Genetic features. An expected 139-bp amplified product was detected in the sample (Fig. 5A). Positively amplified results 
A

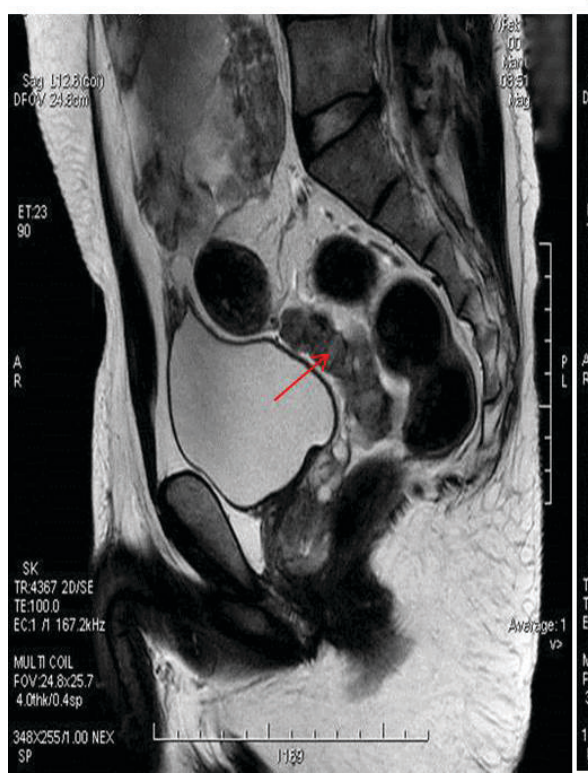

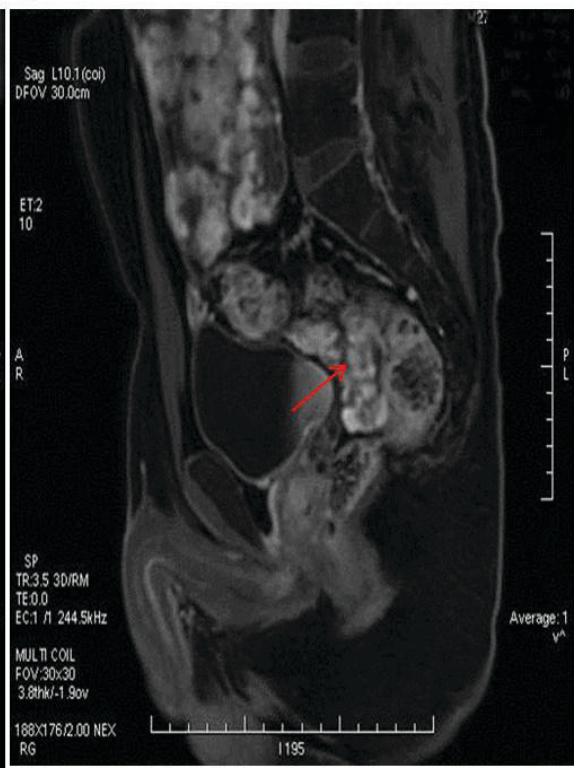

Figure 1. Magnetic resonance imaging examination. (A) The morphology of the tumor was irregular, and the signal was medley consisting of high and low hybrid reinforcement. Tumors are located in the bladder and rectal pit in the lower part of the lower abdomen indicating malignancy, involving the small intestine and rectum. (B) Enhanced scanning image demonstrated the notable inhomogeneous enhancement.

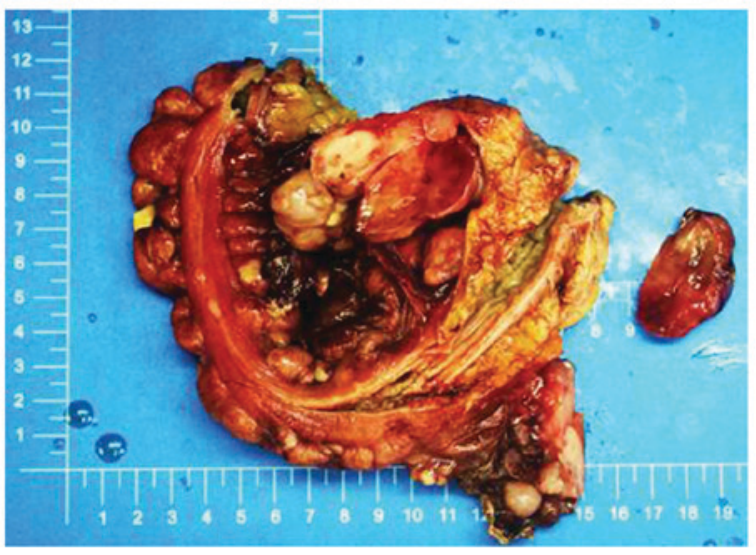

Figure 2. Gross examination depicted a number of masses located in the right abdominal wall, left iliac fossa, the greater omentum, a region of the right hemi-colon, rectum and abdominal cavity. Focal myxomatous appearance was observed.

(268-bp) of $\beta$-actin as a house-holding gene were also present (Fig. 5B). The RANBP2-ALK fusion point was at exon 18 of RANBP2 and exon 20 of ALK (Fig. 5C), which was confirmed by direct sequencing of the chimeric cDNA product.

\section{Discussion}

Clinically, EIMS occurs mainly in children and adolescents, although the overall range in age varies widely, with previous studies reporting on patients aged between 7 months and 65 years, with a median age of 33.4 years (Table II) $(4,12-20)$.

The majority of patients with EIMS are male. In Table II, the male:female ratio is $21: 7$. EIMS is mainly located in abdominal cavity (4). In Table II, 26 cases occurred in the abdominopelvic area $(4,12-20)$, with two cases located in the
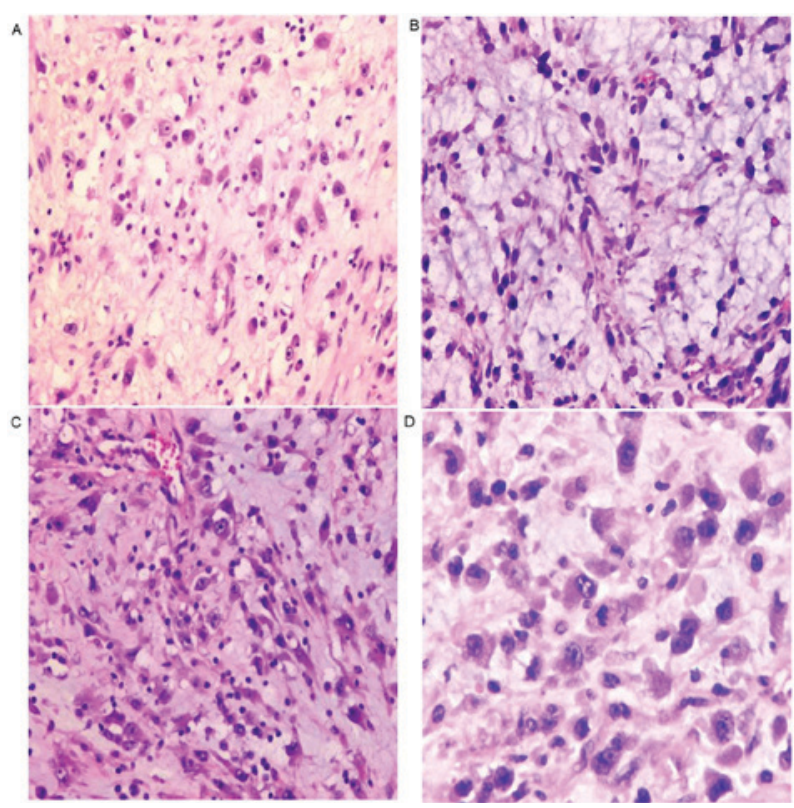

Figure 3. Histopathological examination with haematoxylin and eosin staining. (A) The neoplastic cells exhibit intracellular space between cells (magnification, x200). (B) An abundant myxoid stroma is observed (magnification, x200). (C) Prominent infiltrated lymphocytes are also observed (magnification, x200). (D) Epithelioid tumor cells with eccentric nuclei, large prominent nucleoli and abundant eosinophilic cytoplasm (magnification, $\mathrm{x} 400$ ).

pleural cavity $(21,22)$. The most common symptom of EIMS is abdominal pain prior to surgery (19).

In soft tissue sarcomas, the tumors are often heterogeneous in composition, including areas of fibrosis, mucous degeneration, necrosis, and hemorrhage (23). Magnetic resonance imaging (MRI) enhanced scanning shows that high signal is a rich blood supply area (usually a tumor area), 

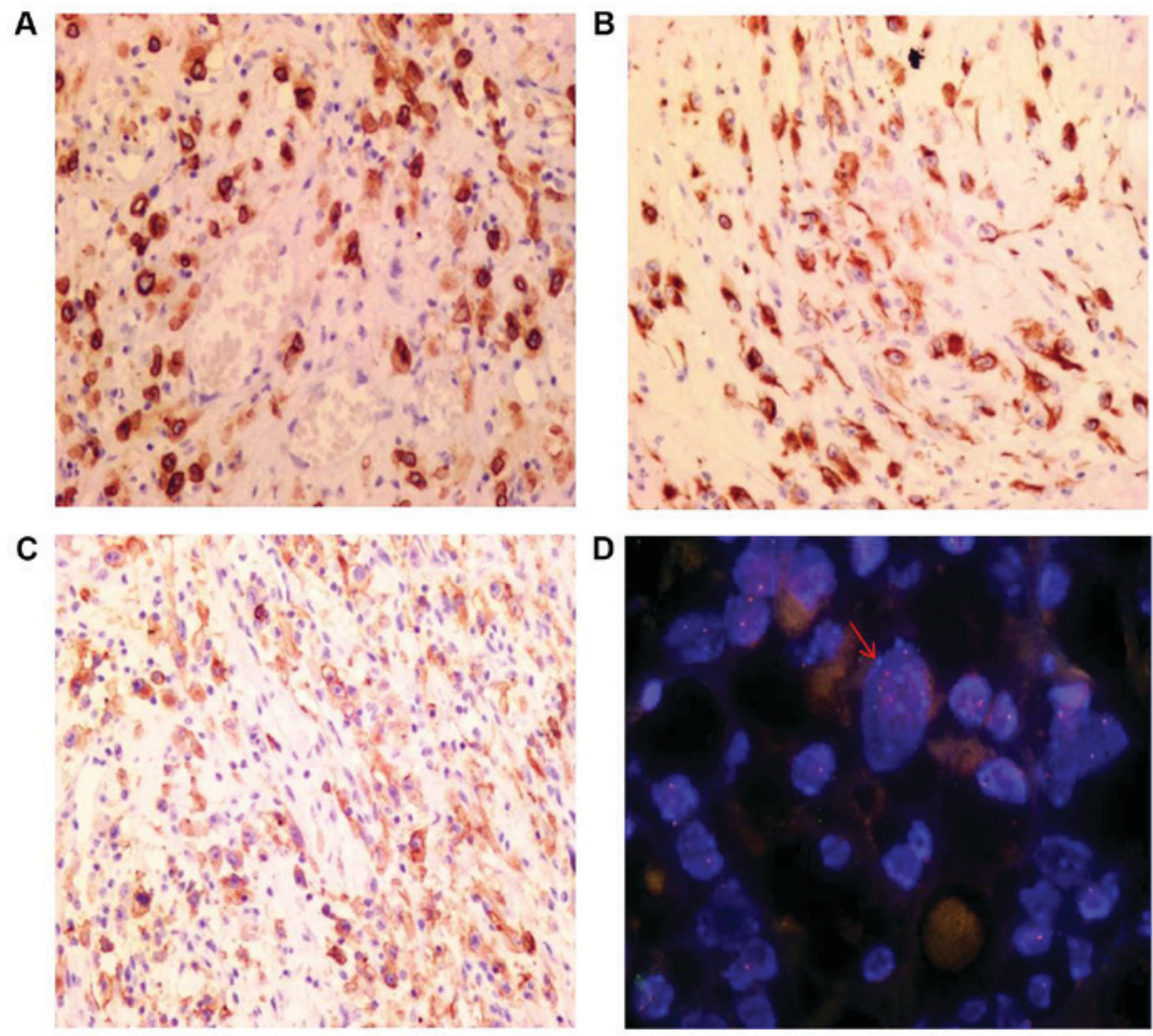

Figure 4. Immunohistochemistry and FISH images. (A) Nuclear membrane staining for ALK (magnification, x200). (B) Cytoplasmic desmin immunostaining (magnification, x200). (C) Cell membrane staining for programmed death-ligand 1 (magnification, x200). (D) FISH indicated a split signal of ALK, confirming the presence of an ALK rearrangement (arrow) (magnification, x1,000). Vysis LSI 3'-ALK Spectrum is orange, Vysis LSI 5'-ALK Spectrum is green. FISH, fluorescent in situ hybridization; ALK, ALK tyrosine kinase receptor.

A

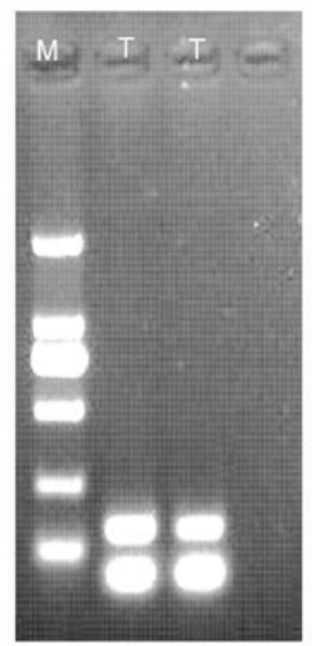

B

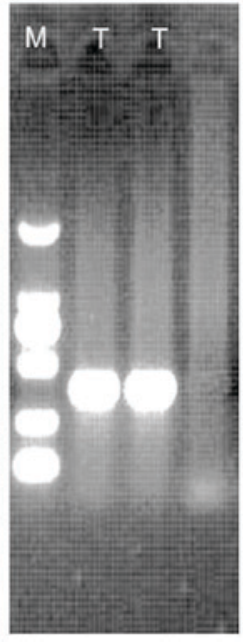

C

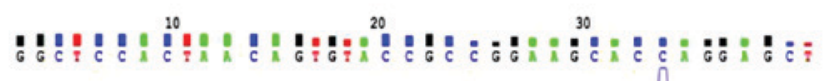

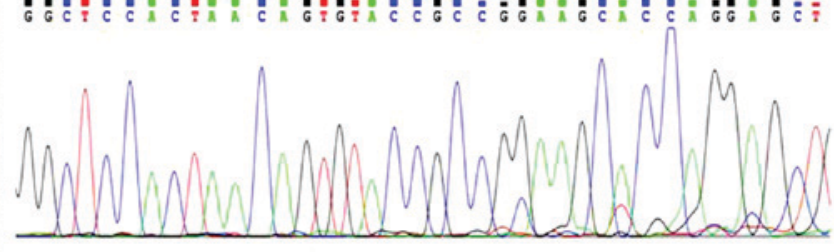

Figure 5. Genetic features of EMIS. (A) RT-PCR with RANBP2 and ALK primers. Lane T: An expected 139-bp product was present in the case. (B) RT-PCR detected the housekeeping gene $\beta$-actin, an expected 268 -bp product was detected. (C) The fusion point of the RANBP2-ALK gene, as indicated by cDNA sequencing, is located between exon 18 of the RANBP2 gene and exon 20 of the ALK gene. M, marker; RT-PCR, reverse transcription-polymerase chain reaction; T, test; RANBP2, RAN binding protein 2; ALK, ALK tyrosine kinase receptor.

no enhancement area is a blood free region (equivalent to a liquefied necrotic area). From high signal to no signal, there also are different levels of reinforcement, which are related to tumor tissue structure, tumor angiogenesis and so on. A tumor can also appear high and low hybrid reinforcement. In the present case, the tumor comprises mucous degeneration and necrosis. The signal was medley consisting of high and low hybrid reinforcement.

Common characteristics of EIMS include: i) Roundto-epithelioid tumor cells; ii) abundant myxoid stroma with inflammatory infiltrates; iii) immunopositivity for ALK with a nuclear membrane or perinuclear staining pattern; iv) 


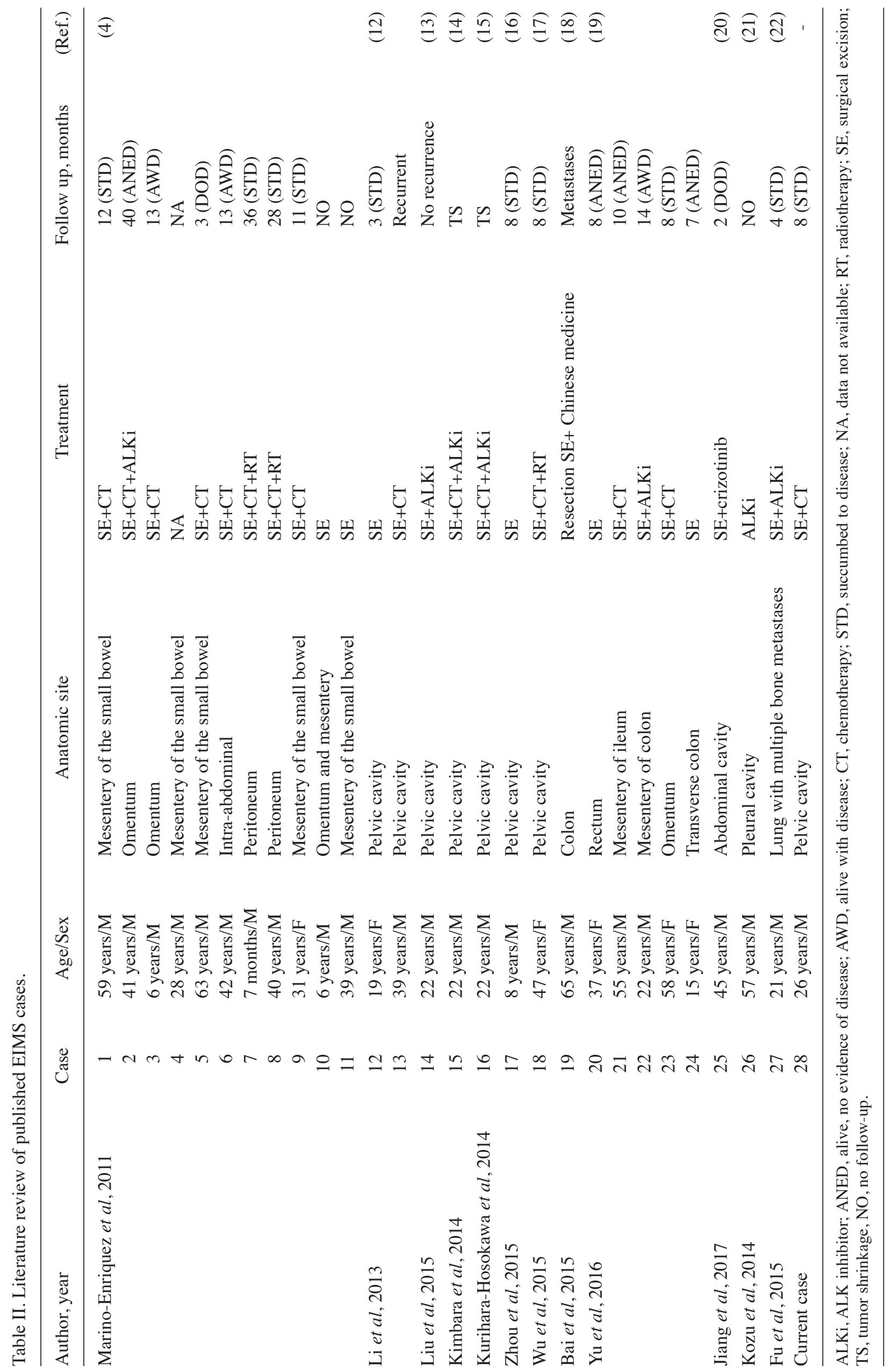




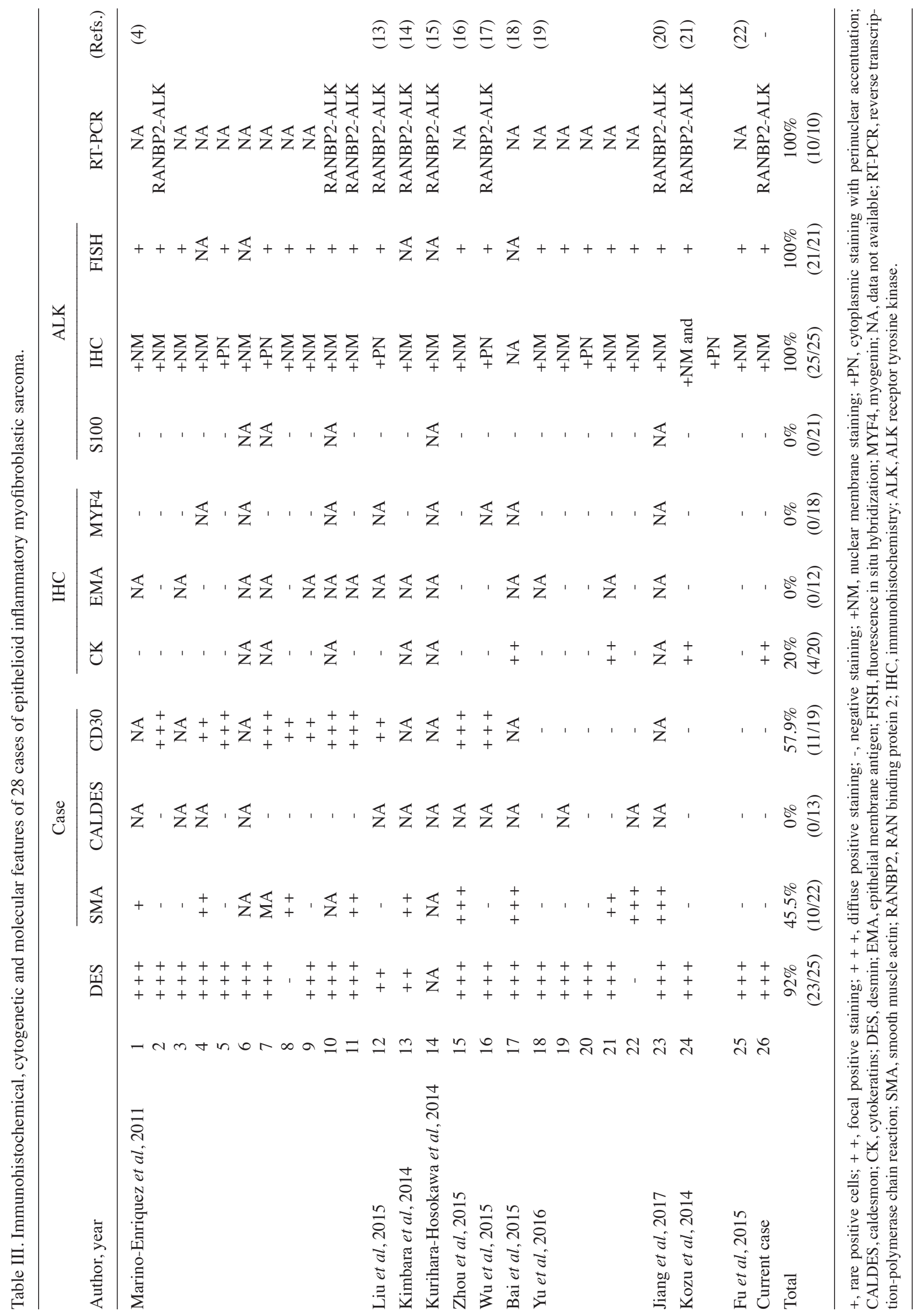


Table IV. Differential diagnosis of EMIS.

\begin{tabular}{lll}
\hline Disease & \multicolumn{1}{c}{ IHC } & \multicolumn{1}{c}{ Molecular pathology } \\
\hline EMIS & CD30 (+), ALK (+), SMA (+), EMA (+) and DES (+) & RANBP2-ALK \\
ALCL & CD30 (+), ALK (+), SMA (+), EMA (+) and DES (-) & T cell gene rearrangement \\
MM & MC (+), CK5 (+), Calretinin (+) and DES (-) & P16/CDKN2A \\
GIST & CD117 (+), DOG1 (+) and CD34 (+) & c-Kit and PDGF $\alpha$ \\
Alveolar Rhabdomyosarcoma & Myogenin (+) & PAX3-FOXO1 and PAX7-FOXO1 \\
& & fusion gene \\
Epithelioid leiomyosarcoma & ALK (-) & HMGA and MED12
\end{tabular}

ALCL, anaplastic large cell lymphoma; MM, malignant mesothelioma; GIST, gastrointestinal stromal tumor; IHC, immunohistochemistry; EMIS, epithelioid inflammatory myofibroblastic sarcoma; RANBP2, RAN binding protein 2; PDGF, platelet growth factor receptor; CK, cytokeratins; CDKN, cyclin-dependent kinase inhibitor; DES, desmin; EMA, epithelial membrane antigen; SMA, smooth muscle actin; PAX, paired box; FOXO1, forkhead box O1; HMGA, high mobility group AT-hook; MED12, mediator complex subunit 12 gene; MC, mesothelial cells.

Desmin ES positive in the cytoplasm of tumor cells; and v) the frequent presence of the RANBP2-ALK fusion gene. All of these features were identified in the present case, meaning a diagnosis of EIMS was reached.

Immunohistochemically, EIMS exhibited nuclear membrane or perinuclear accentuation staining pattern of ALK, which was observed in $100 \%(25 / 25)$ of cases from a selection of previous studies (Table III) (4,13-22). Another diagnostic immunophenotype is the diffuse strong expression of DES in almost all cases (92\%; 23/25). In addition, the tumors displayed variable expression of SMA $(45.5 \%$, $10 / 22)$, CD30 $(57.9 \%, 11 / 19)$ and cytokeratin $(20 \%, 4 / 20)$ (Table III) (4,13-22).

Of the patients with IMT examined by Gleason and Hornick (3), $50 \%$ aberrantly expressed the ALK protein, triggered by clonal rearrangements of the ALK gene located on chromosome 2p23. EIMS tumors harbor a specific RANBP2-ALK fusion gene resulting from $\mathrm{t}(2 ; 2)(2 \mathrm{q} 12 ; 2 \mathrm{p} 23)$. All 21 cases tested by FISH exhibited an ALK translocation signal. Notably, all 10 cases in which the cDNA fusions were examined by RT-PCR exhibited identical fusion points, between exon 18 of RANBP2 and exon 20 of ALK (Table III). RANBP2 encodes a nuclear pore protein, which is likely to lead to the nuclear membrane or perinuclear staining pattern in EIMS.

Diagnosing EMIS can be challenging owing to the unusual epithelioid-to-round cell morphology and atypical nuclear features. EMIS should be differentially diagnosed with diseases as follows: i) Distinguishing EIMS from ALCL can be difficult, as the rare sarcomatoid variant of ALCL can exhibit spindle cell morphology and an overlapping immunophenotype, including reactivity for CD30, ALK and SMA and non-reactivity for EMA. Strong expression of DES and the distinctive nuclear membrane pattern of ALK staining are not observed in ALCL. The RANBP2-ALK has never been reported in ALCL either. ii) Malignant mesothelioma (MM), MC, CK5 and calretinin are present in MM, but ALK is absent. iii) Epithelioid GIST is positive for CD117, DOG1 and CD34. Mutations to $\mathrm{c}-\mathrm{Kit}$ and platelet derived growth factor- $\alpha$ are also present in GIST. GIST exhibits negative ALK staining. iv)
The solid variant of alveolar rhabdomyosarcoma is frequently ALK-positive, lacks fibrovascular stroma and forms sheets of round cells with variable rhabdomyoblastic differentiation. Antibodies against MyoD and myogenin are highly specific and sensitive for its diagnosis; and v) epithelioid leiomyosarcoma (ELS) ELS commonly displays greater cellular atypia and pleomorphism, and higher cellular density. ELS generally lacks an extensive myxoid background and inflammatory infiltrates (12) and lacks ALK nuclear membrane expression (Table IV).

Currently, the majority of cases of EMIS are treated by surgical resection combined with chemotherapy $(4,12)$. Several reports indicated that patients with an ALK gene rearrangement had a notable response to ALK targeted therapy (13-15); however, disease recurrence is common following resection (24). In the present case, the patient was treated with chemotherapy prior to and following surgical resection, but succumbed as a result of cachexia due to tumor metastasis to the thoracic cavity.

Staining for programmed death-ligand 1 (PD-L1) was diffusely positive in the present case. The expression of PD-L1 in this patient was comparable to that in other genitourinary cancer types, such as bladder cancer (20\%) (25), and non-genitourinary cancer types, such as breast cancer (23.4\%) (26), colorectal cancer (36\%) (27), testicular seminomas (73\%) (28) and cancer of the oral cavity (73\%) (29). The PD-1/PD-L1 axis has a notable role in the immune antitumor response $(30,31)$. PD-L1 expression in tumor cells is considered to be predictive of the tumor response to immunomodulatory therapies targeting the PD-1/PD-L1 pathway (32). Unlike chemotherapy and molecularly targeted therapy, the checkpoint blockade immunotherapies result in durable clinical responses through the induction, activation, and expansion of tumor-specific cytotoxic T cells. Immune checkpoints serve an essential role in maintaining self-tolerance and regulating the amplitude and duration of $\mathrm{T}$ cell responses (33). Immunotherapies with checkpoint blockade antibodies that block PD-1 (or its ligand PD-L1) can restore and augment cytotoxic $\mathrm{T}$ cell responses against chemotherapy-refractory tumors, leading to durable responses and prolonged overall survival with tolerable toxicity (33). 
In conclusion, EIMS is a highly aggressive IMT variant with epithelioid-to-round cell morphology, myxoid stroma and nuclear membrane or perinuclear ALK staining. Detection of ALK rearrangement in the present study provides further evidence for the diagnosis of the tumor and a reliable reference for ALK-targeted therapy. The expression of PD-L1 in EIMS indicated the immune checkpoint blockade, which could represent a novel anti-EIMS therapy.

\section{Acknowledgements}

The authors would like to thank to Mr. Yongqi Chen (Department of Pathology, Beijing Aerospace General Hospital, Beijing, China) for editing the language of this paper.

\section{Acknowledgements}

Not applicable.

\section{Funding}

The present study was supported by grants from the 2016 Basic clinical cooperation project of China Capital Medical University (grant no. 3500-11722913).

\section{Availability of data and materials}

The datasets generated in the present study are available on reasonable request from the corresponding author.

\section{Authors' contributions}

$\mathrm{XD}$ was responsible for consulting literature, reviewing slices and drafting the manuscript. YG conducted the molecular genetic studies and immunohistochemistry experiments. HZ made was responsible for the paraffin sections. BL obtained and analyzed the MRI report. WX conducted the FISH experiment. DW took part in analysis and interpretation of data, edited the language of this paper and provided funding support.

\section{Ethics approval and consent to participate}

The study protocol was approved by the Medical Ethics Committee of Beijing Shijitan Hospital, Capital Medical University (Beijing, China).

\section{Consent for publication}

Written informed consent was obtained from patient's father.

\section{Competing interests}

The authors declare that they have no competing interests.

\section{References}

1. Fletcher CDM, Bridge JA, Hogendoorn PCW and Mertens F: World health organization classification of tumors of soft tissue and bone. 4th edition. Lyon, France: IARC Press; pp83-84, 2013.
2. Kirchgesner T, Danse E, Sempoux CH, Annet L, Dragean ChA, Trefois P, Abbes Orabi N and Kartheuser A: Mesenteric inflammatory myofibroblastic tumor: MRI and CT imaging correlated to anatomical pathology. JBR-BTR 97: 301-302, 2014.

3. Gleason BC and Hornick JL: Inflammatory myofibroblastic tumours: Where are we now? J Clin Pathol 61: 428-437, 2008.

4. Mariño-Enríquez A, Wang WL, Roy A, Lopez-Terrada D, Lazar AJ, Fletcher CD, Coffin CM and Hornick JL: Epithelioid inflammatory myofibroblastic sarcoma: An aggressive intra-abdominal variant of inflammatory myofibroblastic tumor with nuclear membrane or perinuclear ALK. Am J Surg Pathol 35: 135-144, 2011.

5. Cook JR, Dehner LP, Collins MH, Ma Z, Morris SW, Coffin CM and Hill DA: Anaplastic lymphoma kinase (ALK) expression in the inflammatory myofibroblastic tumor: A comparative immunohistochemical study. Am J Surg Pathol 25: 1364-1371, 2001.

6. Patel AS, Murphy KM, Hawkins AL, Cohen JS, Long PP, Perlman EJ and Griffin CA: RANBP2 and CLTC are involved in ALK rearrangements in inflammatory myofibroblastic tumors. Cancer Genet Cytogenet 176: 107-114, 2007.

7. Ma Z, Hill DA, Collins MH, Morris SW, Sumegi J, Zhou M, Zuppan C and Bridge JA: Fusion of ALK to the Ran-binding protein 2 (RANBP2) gene in inflammatory myofibroblastic tumor. Genes Chromosomes Cancer 37: 98-105, 2003.

8. Chen ST and Lee JC: An inflammatory myofibroblastic tumor in liver with ALK and RANBP2 gene rearrangement: combination of distinct morphologic, immunohistochemical, and genetic features. Hum Pathol 39: 1854-1858, 2008.

9. Verma S, Turkbey B, Muradyan N, Rajesh A, Cornud F, Haider MA, Choyke PL and Harisinghani M: Overview of dynamic contrast-enhanced MRI in prostate cancer diagnosis and management. AJR Am J Roentgenol 198: 1277-1288, 2012.

10. Hashmi AA, Hussain ZF, Faridi N and Khurshid A: Distribution of Ki67 proliferative indices among WHO subtypes of non-Hodgkin'slymphoma: Association with other clinical parameters. Asian Pac J Cancer Prev 15: 8759-8763, 2014.

11. Livak KJ and Schmittgen TD: Analysis of relative gene expression data using real-time quantitative PCR and the 2(-Delta Delta C(T)) method. Methods 25: 402-408, 2001.

12. Li J, Yin WH, Takeuchi K, Guan H, Huang YH and Chan JK: Inflammatory myofibroblastic tumor with RANBP2 and ALK gene rearrangement: A report of two cases and literature review. Diagn Pathol 8: 147, 2013.

13. Liu Q, Kan Y, Zhao Y, He H and Kong L: Epithelioid inflammatory myofibroblastic sarcoma treated with ALK inhibitor: A case report and review of literature. Int J Clin Exp Pathol 8: 15328-15332, 2015.

14. Kimbara S, Takeda K, Fukushima H, Inoue T, Okada H, Shibata Y, Katsushima U, Tsuya A, Tokunaga S, Daga H, et al: A case report of epithelioid inflammatory myofibroblastic sarcoma with RANBP2-ALK fusion gene treated with the ALK inhibitor, crizotinib. Jpn J Clin Oncol 44: 868-871, 2014.

15. Kurihara-Hosokawa K, Kawasaki I, Tamai A, Yoshida Y, Yakushiji Y, Ueno H, Fukumoto M, Fukushima H, Inoue T and Hosoi M: Epithelioid inflammatory myofibroblastic sarcoma responsive to surgery and an ALK inhibitor in a patient with panhypopituitarism. Intern Med 53: 2211-2214, 2014.

16. Zhou J, Jiang G, Zhang D, Zhang L, Xu J, Li S, Li W, Ma Y, Zhao A and Zhao Z: Epithelioid inflammatory myofibroblastic sarcoma with recurrence after extensive resection: Significant clinicopathologic characteristics of a rare aggressive soft tissue neoplasm. Int J Clin Exp Pathol 8: 5803-5807, 2015.

17. Wu H, Meng YH, Lu P, Ning HY, Hong L, Kang XL and Duan MG: Epithelioid inflammatory myofibroblastic sarcoma in abdominal cavity: A case report and review of literature. Int J Clin Exp Pathol 8: 4213-4219, 2015.

18. Bai Y, Jiang M, Liang W and Chen F: Incomplete intestinal obstruction caused by a rare epithelioid inflammatory myofibroblastic sarcoma of the colon. Medicine (Baltimore) 94: e2342, 2015.

19. Yu L, Liu J, Lao IW, Luo Z and Wang J: Epithelioid inflammatory myofibroblastic sarcoma: A clinicopathological, immunohistochemical and molecular cytogenetic analysis of five additional cases and review of the literature. Diagn Pathol 11: 67, 2016.

20. Jiang Q, Tong HX, Hou YY, Zhang Y, Li JL, Zhou YH, Xu J, Wang JY and Lu WQ: Identification of EML4-ALK as an alternative fusion gene in epithelioid inflammatory myofibroblastic sarcoma. Orphanet J Rare Dis 12: 97, 2017.

21. Kozu Y, Isaka M, Ohde Y, Takeuchi K and Nakajima T: Epithelioid inflammatory myofibroblastic sarcoma arising in the pleural cavity. Gen Thorac Cardiovasc Surg 62: 191-194, 2014. 
22. Fu X, Jiang J, Tian XY and Li Z: Pulmonary epithelioid inflammatory myofibroblastic sarcoma with multiple bone metastases: Case report and review of literature. Diagn Pathol 10: 106, 2015

23. Huang W, Beckett BR, Tudorica A, Meyer JM, Afzal A, Chen Y Mansoor A, Hayden JB, Doung YC, Hung AY, et al: Evaluation of soft tissue sarcoma response to preoperative chemoradiotherapy using dynamic contrast-enhanced magnetic resonance imaging. Tomography 2: 308-316, 2016.

24. Fujiya M and Kohgo Y: ALK inhibition for the treatment of refractory epithelioid inflammatory myofibroblastic sarcoma. Intern Med 53: 2177-2178, 2014

25. Faraj SF, Munari E, Guner G, Taube J, Anders R, Hicks J, Meeker A, Schoenberg M, Bivalacqua T, Drake C and Netto GJ: Assessment of tumoral PD-L1 expression and intratumoral CD8+ T cells in urothelial carcinoma. Urology 85: 703.e1-e6, 2015.

26. Muenst S, Schaerli AR, Gao F, Däster S, Trella E, Droeser RA, Muraro MG, Zajac P, Zanetti R, Gillanders WE, et al: Expression of programmed death ligand 1 (PD-L1) is associated with poor prognosis in human breast cancer. Breast Cancer Res Treat 146: 15-24, 2014.

27. Droeser RA, Hirt C, Viehl CT, Frey DM, Nebiker C, Huber X, Zlobec I, Eppenberger-Castori S, Tzankov A, Rosso R, et al: Clinical impact of programmed cell death ligand 1 expression in colorectal cancer. Eur J Cancer 49: 2233-2242, 2013.
28. Fankhauser CD, Curioni-Fontecedro A, Allmann V, Beyer J, Tischler V, Sulser T, Moch H and Bode PK: Frequent PD-L1 expression in testicular germ cell tumors. Br J Cancer 113: 411-413, 2015.

29. Straub M, Drecoll E, Pfarr N, Weichert W, Langer R, Hapfelmeier A, Götz C, Wolff KD, Kolk A and Specht K: CD274/PD-L1 gene amplification and PD-L1 protein expression are common events in squamous cell carcinoma of the oral cavity. Oncotarget 7: 12024-12034, 2016.

30. Zandberg DP and Strome SE: The role of the PD-L1: PD-1 pathway in squamous cell carcinoma of the head and neck. Oral Oncol 50: 627-632, 2014.

31. Patel SP and Kurzrock R: PD-L1 expression as a predictive biomarker in cancer immunotherapy. Mol Cancer Ther 14: 847-856, 2015

32. Berger R, Rotem-Yehudar R, Slama G, Landes S, Kneller A, Leiba M, Koren-Michowitz M, Shimoni A and Nagler A: Phase I safety and pharmacokinetic study of CT-011, a humanized antibody interacting with PD-1, in patients with advanced hematologic malignancies. Clin Cancer Res 14: 3044-3051, 2008.

33. Ma W, Gilligan BM, Yuan J and Li T: Current status and perspectives in translational biomarker research for PD-1/PD-L1 immunecheckpoint blockade therapy. J Hematol Oncol 9: 47, 2016. 